ACCEPTED MANUSCRIPT

\title{
Summary of the 3rd IAEA technical meeting on divertor concepts
}

To cite this article before publication: Matteo Barbarino et al 2020 Nucl. Fusion in press https://doi.org/10.1088/1741-4326/ab9a0c

\section{Manuscript version: Accepted Manuscript}

Accepted Manuscript is "the version of the article accepted for publication including all changes made as a result of the peer review process, and which may also include the addition to the article by IOP Publishing of a header, an article ID, a cover sheet and/or an 'Accepted Manuscript' watermark, but excluding any other editing, typesetting or other changes made by IOP Publishing and/or its licensors"

This Accepted Manuscript is @ 2020 IAEA, Vienna.

During the embargo period (the 12 month period from the publication of the Version of Record of this article), the Accepted Manuscript is fully protected by copyright and cannot be reused or reposted elsewhere.

As the Version of Record of this article is going to be / has been published on a subscription basis, this Accepted Manuscript is available for reuse under a CC BY-NC-ND 3.0 licence after the 12 month embargo period.

After the embargo period, everyone is permitted to use copy and redistribute this article for non-commercial purposes only, provided that they adhere to all the terms of the licence https://creativecommons.org/licences/by-nc-nd/3.0

Although reasonable endeavours have been taken to obtain all necessary permissions from third parties to include their copyrighted content within this article, their full citation and copyright line may not be present in this Accepted Manuscript version. Before using any content from this article, please refer to the Version of Record on IOPscience once published for full citation and copyright details, as permissions will likely be required. All third party content is fully copyright protected, unless specifically stated otherwise in the figure caption in the Version of Record.

View the article online for updates and enhancements. 


\title{
divertor concepts
}

Summary of the 3rd IAEA technical meeting on

\author{
Matteo Barbarino ${ }^{1}$, Anthony Leonard ${ }^{2}$, Nobuyuki N. Asakura ${ }^{3}$, Marcin \\ Jakubowski ${ }^{4}$, Masahiro Kobayashi ${ }^{5}$, Bruce Lipschutz ${ }^{6}$, Rudolf Neu ${ }^{7}$, Liang Wang ${ }^{8}$, \\ Marco Wischmeier ${ }^{7}$
}

${ }^{1}$ International Atomic Energy Agency, Vienna International Centre, PO Box 100, 1400 Vienna, Austria

${ }^{2}$ General Atomics, San Diego, CA, United States of America

${ }^{3}$ National Institutes for Quantum and Radiological Science and Technology, Naka, Ibaraki 311-0193, Japan

${ }^{4}$ Max-Planck Institute for Plasma Physics, Wendelsteinstrasse 1, 17491 Greifswald, Germany

${ }^{5}$ National Institute for Fusion Science, 322-6 Oroshi-cho, Toki-shi 509-5292, Japan

${ }^{6}$ York Plasma Institute, Department of Physics, University of York, York YO10 5DD, United Kingdom

${ }^{7}$ Max-Planck Institute for Plasma Physics, Boltzmannstr.2, D-85748 Garching, Germany

${ }^{8}$ Institute of Plasma Physics, Chinese Academy of Sciences, Hefei 230031, People's Republic of China

E-mail: M.Barbarino@iaea.org

Received xxxxxx

Accepted for publication $\mathrm{xxxxxx}$

Published xxxxxx

\begin{abstract}
This report summarizes the contributions presented at the 3rd IAEA technical meeting on divertor concepts, held in Vienna, Austria, 4-7 November 2019. The meeting brought together more than 70 experts from nuclear fusion research sites worldwide to discuss the different aspects that the divertor design and fusion machine operation involve, from ITER divertor developments to innovative technologies for future DEMO divertor. The main topics of the meeting were: divertor and confinement; radiative power exhaust; scrape-off layer (SOL) and divertor physics; steady state operation and transient heat loads; plasma facing components materials and heat exhaust for steady state operation; and divertors for DEMO and future power reactors.
\end{abstract}

Keywords: divertor and confinement, radiative power exhaust, scrape-off layer and divertor physics, steady state operation and transient heat loads, plasma facing components materials and heat exhaust for steady state operation, divertors for DEMO and reactors

\section{Introduction}

The main objective of fusion technology development is to deliver a safe, economical, essentially unlimited and emissionfree energy source. While considerable progress has been made during the last few years, there is still a great deal of research and development needed.

The International Atomic Energy Agency (IAEA) fosters international collaboration, coordination and exchange of scientific and technical results to help close the existing gaps in physics, technology and safety with the objective of encouraging the development of new technologies and science for ITER and facilitating the coordination of the global effort for the development of future fusion power plants.

IAEA's technical activities in support of fusion research take place under the guidance of the International Fusion Research Council and include but are not limited to plasmaengineering aspects, divertor design and fusion machine operation. 
Journal Title

The 3rd IAEA Technical Meeting on Divertor Concepts was held in Vienna, Austria, 4-7 November 2019 [1]. Presentations are available through the IAEA website at https://nucleus.iaea.org/sites/fusionportal/Shared\%20Docum ents/DC\%202019\%20Material.pdf.

Previous meetings in this series were held in Vienna, Austria (2015) [2] and Suzhou, China (2017) [3], and proved successful for discussing open physics and technology issues associated with divertors and fostering discussions on solutions for different power exhaust problems. In addition, a very first IAEA Technical Committee Meeting on Divertor Concepts was held in Aix-en-Provence, France (2001) [4].

The 2019 meeting was a unique occasion to discuss divertor physics in various magnetic configurations, novel ideas for next step devices and, most importantly, the ITER divertor the largest and most complex divertor ever constructed. Although this design reflects current understanding and technology, power exhaust requirements for future fusion power plants need further development to satisfy the relevant engineering constraints.

73 experts from 17 Member States (Austria, China, Czech Rep., Egypt, France, Germany, Italy, Japan, Lithuania, Nepal, Netherlands, Republic of Korea, Russian Federation, Switzerland, Ukraine, United Kingdom, Unites States) and 1 International Organization (ITER Organization) presented the most prominent results on plasma physics and engineering aspects in technology design and fusion machine operation, from ITER divertor developments to innovative technologies for DEMO divertor.

\section{Topics and session summaries}

There were 73 contributions, 32 orals and 41 posters, on the following topics:

- Plasma facing component (PFC) design;

- Divertor plasma control;

- Implications of applied three-dimensional (3D) fields;

- Stellarator divertors;

- Alternative materials for PFCs;

- Core-boundary plasma compatibility;

- DEMO divertors design;

- Divertors in next step devices.

The scientific programme and paper selection were the responsibility of an International Programme Advisory Committee composed of the following members: Anthony Leonard (Chair, United States), Nobuyuki Asakura (Japan), Suk-Ho Hong (Republic of Korea), Masahiro Kobayashi (Japan), Brian LaBombard (United States of America), Bruce Lipschultz (United Kingdom), William Morris (United Kingdom), Rudolf Neu (Germany), Richard Pitts (ITER
Organization), Liang Wang (China), Marco Wischmeier (Germany), Guoyao Zheng (China).

Brief summaries of the contributions and corresponding round table discussions are presented in the following topical sections.

\subsection{PFC design}

This session (chaired by L. Wang) highlighted recent progress and issues in PFC designs for DEMO.E. Visca (Italy) presented EU DEMO reactor designs for water-cooled divertor target concepts. The water-cooled W/Cu monoblock structure is acknowledged to be the most promising divertor target concept for present superconducting tokamaks and fusion reactors. The presentation proposed that the flat tile $\mathrm{W}$ with more cooling capability for power exhaust should be given more attention for future application. In addition, the manufacture of tungsten R\&D, together with active cooling, e.g. 3D printing needs to be further developed and optimized. On the testing side, PFC experiments on the tokamaks, especially the hotspots of $\mathrm{W}$, its erosion and edge damage, including maintainability in the tokamak environment needs additional validation. More modelling work for future prediction needs to be carried out. In development of active water-cooled W divertor PFCs, European labs are able to produce advanced $\mathrm{W}$ divertor water-cooled PFCs for EU DEMO application. These components withstand up to $20 \mathrm{MW} / \mathrm{m}^{2}$ cyclic heat load. Standard mock-up geometry is required to enable concept comparison. Important $R \& D$ progress for novel technologies (i.e. W/Cu composites for heat-sink and pipes, FGM interlayer, new ENEA-HRP furnace) was presented. Open issues for further investigation were interlayer thickness (is $1 \mathrm{~mm}$ really necessary?), standards and codes for novel materials of pipes $\left(\mathrm{W}_{\mathrm{f}}-\mathrm{Cu}\right)$, neutron damage on materials, possible scale-up of selected concepts, and performances under long-pulse thermal loads.

Progress on helium-cooled divertor target concepts for EU DEMO was presented by B.-E. Ghidersa (Germany). Heliumcooled divertor concepts are still being developed for designs applicable for fusion reactors, and these have not yet been applied in any existing tokamaks. A new helium multi-jet pipe target concept designed for EU-DEMO was presented, with a heat flux tolerance of $8 \mathrm{MW} / \mathrm{m}^{2}$ (83h) for 1000 pulses and $10 \mathrm{MW} / \mathrm{m}^{2}(2 \mathrm{~h})$ for 25 pulses. The design can be integrated into a EU-DEMO target plate. Further steps will be made on the metallographic analysis of the mock-ups, setting-up a manufacturing path for the current design, and experimental evaluation of cooling performances at design conditions (KATHELO: $500^{\circ} \mathrm{C}, 10 \mathrm{MPa}$ ).

M. Firdaouss (France) presented experimental results from WEST on the application of $\mathrm{W} / \mathrm{Cu}$ monoblock in its divertor. The presentation focused on damage of W PFC units observed after the experimental campaigns. It was not obvious what caused the damage of the ITER-like W PFC edges under the 
conditions of moderate heat flux. Cracks and local melting on misaligned Plasma Facing Unit (PFU) sides (both sharp and chamfered) were presumably linked to transients outside the strike zone. Optical hot spots and subsequent damage was observed, even for PFUs aligned within specifications. The hot spots could not be avoided for the current design. It was concluded that PFU misalignment tolerances must be defined to constrain the manufacturing and assembly process, as specified for the ITER divertor design.

An additive manufacturing technique of $\mathrm{W}$ by means of laser powder bed fusion (LPBF) for PFC applications was presented by A. von Müller (Germany). The 3D components are created by sequential layer-wise deposition of material under computer control with more or less arbitrary shape production. It is shown that by means of LPBF pure $\mathrm{W}$ with relative mass density $\sim 98 \%$ can be consolidated, in a high temperature process with substrate temperatures up to $1000^{\circ} \mathrm{C}$. However, the material consolidated with high relative mass densities exhibits formation of micro-crack defects. Also, the laser power density and the laser scanning speed influence the quality of the additively manufactured part. In the next step, additive manufacturing of "complex" W structures with porous W lattice structures is planned for limiter applications.

Discussion was organized around the following points raised in this session: (i) cassette monoblock structure and flattype structure of PFCs; (ii) water-cooled W-Cu versus heliumcooled $\mathrm{W}-\mathrm{Cu}$ technologies; (iii) how to minimize the misalignment, structure design \& installation; (iv) W divertor lessons learned from present tokamaks; (v) material degradation due to neutron irradiation; and (vi) modelling and analysis validation to support the design.

For cassette monoblock structures and flat-tile structures for PFCs the discussion focus was on their structure and shape. The monoblock structure is good but needs strong cooling and could suffer from critical heat fluxes. Flat-tiles can achieve higher thermal efficiency and should be studied in more detail. Some devices should examine the flat-tile. If some effort is made to improve the $\mathrm{W}$ bonding technology, that research can be done more directly.

The discussion then turned to water-cooled $\mathrm{W}-\mathrm{Cu}$ versus helium-cooled W-Cu technologies. Water cooled concepts have proven successful in existing devices but would be challenging for a reactor environment. For water versus helium, the issue is the sufficient heat flux removal. If heat loads can be kept low $\left(\leq 10 \mathrm{MW} / \mathrm{m}^{2}\right)$, He can be the preferable option. Conservative designs are still being considered, given for example, the uncertainty of tungsten ductility. As long as DEMO maximum power loads are not known, water-cooled components will remain the baseline for its design. However, He-cooled PFCs are still considered and should be qualified in the next 4-6 years. Further design work for reactors is needed, since they will be operated at a high temperature for low tritium retention.

Minimization of misalignment of structures in design and installation remain an important aspect for the DEMO divertor. However, this engineering issue was not discussed in detail during the meeting since most of the participants focused on physics issues. Alignment tolerance remains a very important issue for PFC design and applications.

Subsequently, lessons on $\mathrm{W}$ divertors learned from present day tokamaks were discussed. As mentioned before experimental results on WEST W components highlighted some challenges. However, reasons for the $\mathrm{W}$ monoblock damage were not yet clear emphasizing the need to assemble results from different devices with W PFCs. It is suspected that misalignment of the W monoblock caused part of the damage observed in WEST and EAST, whereas ASDEX Upgrade (AUG) and JET do not exhibit such severe problems. Although in the case of WEST the X-point is much closer to the target compared to the other machines it was reported that according to investigations this should not be the reason for the damage. Therefore, it was speculated that the damage at the monoblock edges may have been caused by strong transient events such as disruptions and runaways (edge localized modes (ELMs) can be excluded because no stable $\mathrm{H}$ mode has yet been achieved in WEST). In the next campaign in WEST, specific emphasis will be put on the alignment. Due to the large magnetic ripple WEST always will have locally intense power fluxes, and the question remains whether it can live with these inevitable hot spots.

Material degradation due to neutron irradiation was briefly discussed. The neutron irradiation is estimated to cause 4 displacements per atom (dpa) for $\mathrm{W}$ and $\sim 13 \mathrm{dpa}$ for $\mathrm{CuCrZr}$ cooling tubes during the expected lifetime of a W-Cu divertor PFC in DEMO. However, the neutron induced degradation is still very much an uncertain issue.

The final discussion was on modelling and analysis validation to support design. $\mathrm{W}$ erosion is of the most importance to bring forward, with the thickness of $\mathrm{W}$ and the surface temperature needed to be taken into account. An armour thickness of $8 \mathrm{~mm}$ seems to provide sufficient margin under low erosion conditions and for the thermal baffling of the heat sink from slow transient events. In steady state, detached plasma conditions almost no erosion is expected. However, even for semi-detached plasmas there should be little erosion. ELMs can complicate this issue and an $8 \mathrm{~mm}$ armour thickness could be marginal.

Specifically, ELMs could strongly increase the erosion rate and their control is mandatory not only to prevent damage by cracking and melting. It needs to be noted that for modelling the erosion, gross erosion alone is not appropriate and redeposition must be taken into account. If $90 \%$ of eroded $\mathrm{W}$ is redeposited, there should be no problem. Here, the divertor plasma temperature, $\mathrm{T}_{\mathrm{e}}$ and $\mathrm{T}_{\mathrm{i}}$, is a critical factor but 
Journal Title

simulation and theory cannot predict these parameters with sufficient accuracy for ITER. Even more so, the size of tolerable ELMs needs to be examined further. Specifically, the energy of impinging particles during transients needs also to be studied. In terms of redeposition, the behaviour after melting is also not clear. Summarizing, more data in steadystate tokamaks are needed before finalizing designs for DEMO.

\subsection{Divertor plasma control}

This session (chaired by B. Lipschultz) addressed two general questions: (i) control of the detachment as it develops; and (ii) control of detachment characteristics. Control of detachment potentially includes many aspects of detachment from heat loads on the target and impurity compression in the divertor (including $\mathrm{He}$ ) to effects on the core plasma (e.g. impurity levels and energy confinement). Whereas, control of detachment characteristics is aimed at divertor design including the geometry/location/material of surfaces (strike point angle to the surface, closure of the divertor) and the magnetic topology (poloidal and total field flux expansion). The above are broad areas and given the limited time and number of presentations on the control subject, the coverage of these presentations was small. The following oral contributions were given:

- "The small angle slot divertor concept for steady-state fusion" by H. Guo (United States);

- "Using variations in divertor magnetic topology and geometry to optimize divertor detachment" by B. Lipschultz (United Kingdom);

- "Latest advances in active control of H-mode detachment and its physics on EAST for ITER/CFETR" by L. Wang (China); and

- "X-point radiation and detachment control in ASDEXUpgrade" by M. Bernert (Germany).

On control of detachment, it was shown that control of radiation at and above the X-point after detachment was successful at AUG, using the $\mathrm{N}_{2}$ seeding gas feed as the actuator to control the position of the $\sim 2 \mathrm{~cm}$ region of peaked radiation. In addition, M. Bernert (Germany) showed that first measurements of the effect of the radiation/and seeding on the core plasma profiles was significant, but the overall energy confinement was only degraded by $\sim 10 \%$.

The presentation by L. Wang (China) on EAST emphasized control of detachment through target probe and radiation (below the X-point) measurements, again utilizing $\mathrm{N}_{2-}$ seeding. The two control methods were even combined for some amount of discharges on EAST. The radiation control was utilized with success on DIII-D as well.
On control of detachment characteristics, presentations highlighted the importance of strike point angle (the so-called 'vertical' vs 'horizontal' target where the former leads to lower detachment threshold), baffling to trap neutrals (which raises the divertor density and lowers temperature, bringing on detachment) as well as poloidal and total flux expansion (moving the target to lower total magnetic field which lowers the parallel heat flux and reduces the detachment threshold in a SOLPS study). The DIII-D results presented by $\mathrm{H}$. Guo (United States) compared the various available closure and strike point angles available and found consistency with past work on lowering the detachment threshold; there appeared to be additional advantages to some of the variations in terms of the core confinement depending on $B \times \nabla B$ direction (best when the $B \times \nabla B$ direction towards the active X-point. The SOLPS studies of TCV presented by B. Lipschultz (United Kingdom) showed that a quantitative assessment of various baffling and strike point angle choices could be made, in terms of the fraction of neutral source from the target ionized in a flux tube near the separatrix.

Some of the considerations made during the time dedicated to discussion are given below:

- It seems unlikely that all detachment characteristics (including effect on the core) can be controlled simultaneously. Rather, one would control 1-2 characteristics and monitor the others to make sure some boundary was not exceeded.

- Certainly, a divertor characteristic that one wants to maintain is to keep the He ash removal equal to the source rate so that the He core fraction does not significantly lower the fusion power rate (e.g. a $5 \% n_{\mathrm{He}} / n_{\mathrm{e}}$ concentration leads to a $20 \%$ reduction in fusion power). The He concentration in the divertor might be followed indirectly through neutral measurements (RGA) with slow time scales. It is probably easier to measure the core concentration.

- There was concern expressed about radiation from near a $\mathrm{X}$-point or strike point which in proximity to a surface could lead to localized heat loads. This is probably a matter of divertor design other than being specific to control. But certainly, heat loads (in theory) can be monitored as part of divertor plasma control.

- A question was asked whether radiation can be made to spread along the divertor leg more than presently. For a given impurity the range in $T_{\mathrm{e}}$ of the high emissivity is often limited (e.g. 5-60 eV for C) so that one can only extend the radiation region by reducing the $\nabla T$ along the field (e.g. lowering q\| or through total flux expansion).

In addition, the topic of extrapolability from AUG to a DEMO of a successfully stable (in position, intensity, control) $\mathrm{X}$-point radiator was brought up. However, for JET, good stability was only achieved at high $P_{\mathrm{SOL}} / P_{\mathrm{LH}}$. The 
understanding to a deeper level than now of whether an Xpoint radiator makes sense will require a combination of pedestal stability and radiation (and $T_{\mathrm{e}}$ ) profiles.

The ideal detachment would be one that is passively stable and also is a large 'shock absorber' for transients in heat loads, density, and impurity seeding faults. For example, a detachment developed during a $P_{\text {SOL }}$ transient could absorb all the additional power without reattaching. For the above-the$\mathrm{X}$-point radiator one would need to study what amount of additional power could be absorbed and stay detached. Same for the Super-X or total flux expansion where predictions indicate that a large range of $q \|$ change can be absorbed with a movement of the detachment front.

The optimal divertor size with respect to the size of the core plasma was also discussed. This will likely require modelling studies to be able to determine the effects of leg length (and where the detachment front is) on pumping, power removal, 'shock-absorbing' capability, and effects on the core plasma, among others.

Finally, there was general agreement on the need of a survey on the range of transport coefficients utilized in SOL/divertor codes and whether they change in the divertor and how sensitive the solution is to their choice.

\subsection{Implications of applied 3D fields}

This session (chaired by M. Jakubowski) highlighted the implications of applied 3D fields. The ITER divertor has been designed for axisymmetric configurations, yet symmetry breaking resonant magnetic perturbations (RMPs) will be applied for control of ELMs. Applying external field breaks the axisymmetry of the plasma boundary in tokamaks. M. Jakubowski (Germany) and O. Schmitz (United States) showed that with RMP fields applied, a striation of/the divertor target heat and particle flux pattern is detected, which proves the existence of helical magnetic fingers reaching from the X-point outward to the divertor target. In a diverted tokamak, poloidal magnetic flux escaping the outer edge of this stochastic region is organized by complex topological structures known in nonlinear dynamical systems theory as homoclinic tangles [5]. Such a tangle is generated by a splitting of the separatrix into a set of invariant manifolds when perturbed by small non-axisymmetric magnetic fields, which appear as a number of external lobes at the separatrix [6]. As a result, 3D plasma boundary is formed, which potentially alters the divertor and transport characteristics at the plasma edge [7]. For the reactor-scale fusion devices such as ITER or DEMO, control of the divertor target power loading, both in steady state and during ELMs, is particularly challenging with regard to tungsten target lifetime. It should preferably be reduced below a certain value so that the divertor target cooling capability ensures a planned long-term replacement period for the targets. It is widely accepted that RMPs can be an effective method to achieve this since the peak heat flux is expected to be substantially reduced due to the profile broadening while ELMs are being suppressed or significantly mitigated [8].

H. Frerichs et al. in [9] investigated the influence of RMPs on a number of alternative configurations foreseen for NSTX$\mathrm{U}$, which are also possible candidates for a DEMO tokamak. This includes a number of snowflake configurations. Advanced configurations, e.g. snowflake plus or snowflake minus show typically higher number of external lobes as compared to the standard poloidal divertor. One should note that the asymmetric snowflake minus divertor shares properties with an $\mathrm{X}$-divertor, i.e. the snowflake minus configuration does fulfil the geometric properties of an Xdivertor for the outer divertor leg, if the secondary X-point is located just behind the divertor plates. In this configuration, strong corrugation of the separatrix at the outer legs leads to a dense distribution of the lobes at the outer leg. With sufficiently high anomalous diffusion, the power flux would not be striated, instead broad strike lines would appear on the divertor target.

Recently, the numerical capability to investigate the predicted detached divertor scenario at ITER with such 3D deformations has been made available. H. Frerichs (United States) presented modelling results for the ITER baseline discharge with $n=3$ RMP fields applied. Those simulations also included the plasma response obtained with the single fluid resistive magnetohydrodynamics (MHD) code MARSF. Strong screening response is reported in the calculations, but plasma response includes field amplification near the separatrix. As a result, screening of most of the resonances leads to a narrower region of broken flux surfaces, but radial extension of the divertor footprint occurs due to field amplification near the separatrix. Detachment transition with the RMP occurs at a lower gas puff rate and lower peak particle flux at the original strike zone, consistent with a lower upstream heat flux that it connects to along the 3D SOL. However, a secondary non-axisymmetric strike location exists radially further outward, which remains attached because of a magnetic connection to higher upstream temperatures further inside the bulk plasma, carrying significant heat fluxes to this previously low flux domain in the divertor. This strongly reduces the potential for complete power dissipation, and it is a new feature in the ITER divertor revealing a challenge for the integration of RMP ELM control with a feasible divertor operation regime.

In KSTAR, as presented by H.H. Lee (Republic of Korea), dynamics of the outer divertor heat flux are being comprehensively investigated. In particular, modification of the power load distribution during the RMP ELM-suppressed phase has been discussed. In KSTAR, 3-row and 4 column invessel control coils (IVCCs) are used to apply $n=1$ or $n=2$ magnetic perturbations with various toroidal phasing and different configurations. Recently improved degree of control 
Journal Title

Journal XX (XXXX) XXXXXX https://doi.org/XXXX/XXXX

of the RMPs coils made possible investigating the dynamics of the divertor heat flux with changing toroidal phase of the RMPs during a single plasma discharge while continuously maintaining the ELM suppression [10]. It was found that distribution of the divertor heat flux becomes threedimensional with striation patterns closely resembling results of the field line tracing calculation, especially when including plasma response [11]. It is a common feature of KSTAR RMP discharges that the inter-ELM heat flux at the outer divertor target is higher as compared to the inter-ELM phase with no RMPs. This observation contradicts the expectation based on the EMC3-EIRENE calculation. The underlying physics of the phenomenon is under investigation on KSTAR. The hypothesis is that RMP leads to density pump-out, which results in reduced amount of radiated power and thus to an increase of the divertor loads. In addition, it was demonstrated that intentionally misaligned ITER-like 3-row RMPs cannot only suppress Type-I ELMs, but also disperse divertor heat fluxes in a wider area reducing the peak heat flux, while minimizing electromagnetic loads on the RMP coils. Along with the study on the RMPs configuration optimization, the methodology to obtain high-density plasma ensuring RMP ELM-suppressed regime compatible with detached divertor was ardently searched and some promising results were achieved in KSTAR.

Finally, during the discussion session, the following points were raised:

- Use of RMP coils in DEMO tokamak. R. Kembleton (United Kingdom) discussed activities of the EUROfusion DEMO group concerning ELM mitigation scenarios. At the moment a consistent plasma scenario that avoids ELMs does not exist. It is, however, acknowledged as an issue for DEMO divertor. Currently alternative plasma scenarios such as QH- and I-mode are being investigated, although these clearly have a much-reduced physics basis than ELMy H-mode. Currently, RMP ELM-mitigation coils are not considered as a solution for DEMO, although some work has taken place in the past to approach this issue.

- Use of RMP coils in ITER. M. Jakubowski stressed that in present day devices, it is very challenging to achieve both: full ELM mitigation and detached outer divertor. ITER will operate at much higher separatrix densities, while keeping very low pedestal collissionality, as compared to DIII-D, AUG or KSTAR. There is an activity within ITPA-DSOL group to try to study both experimentally and numerically such scenarios. Recently KSTAR showed some progress towards ELM suppression and mitigation at higher plasma density. Results of EMC3-EIRENE showed that ITER may need to deal with higher than expected values of peak heat flux at the secondary strike line. C.S. Chang (United States) indicated that the edge specialized XGC code, which includes the magnetic separatrix and X-point together with neutral particle recycling in realistic divertor geometry, predicts that the edge pedestal in the full-current ITER will be at least twice wider than the MHD-limited (peelingballooning) pedestal width.

- It was asked if SOL/divertor radial transport changes at ELM suppression onset. Pedestal transport obviously changes as shown by density pump-out, but does this change extend into the field lines connecting to the target? KSTAR data seems to hint to transport changes. Understanding this transport will be critical for predictive models for control of the divertor heat flux during application of 3D fields for ELM control. In response, increased priority for studying this issue in existing experiments was called for.

\subsection{Stellarator divertors}

This session (chaired by M. Kobayashi) was dedicated to stellarator divertors. R. Koenig (Germany) and M. Kobayashi discussed divertor optimization toward stellarator reactor based on the experimental observations and on the modelling results in W7-AS/X and LHD. While these devices have significantly different magnetic geometry from each other, that is, W7-AS/ $\mathrm{X}$ is an island divertor and LHD is a helical divertor, common physics aspects and different observations of the divertor performance were presented and discussed throughout the session.

In W7-AS/X and LHD, it was recognized that the crossfield transport is important in the energy, particle, and the momentum transport in the divertor region due to the long field line connection length. In W7-AS and LHD, this feature appears as an absence of a high-recycling regime due to the loss of plasma momentum via cross-field interaction between counter-streaming flow formed in the 3D flux tubes. In W7$\mathrm{X}$, on the other hand, the high-recycling regime was confirmed where the larger island size avoids the cross-field interaction of the plasma flows. The high-recycling regime, which is well established in tokamaks, provides high neutral compression in the divertor plenum and thereby foresees high pumping efficiency in W7-X in upcoming experiments with divertor cryo-pumps. In LHD, the divertor neutral compression was improved by a factor of $\sim 10-15$ by closing the divertor structure. The newly developed divertor cryo-sorption pump demonstrated at least $50 \%$ pumping efficiency. Further upgrade of the pumping capability is ongoing to achieve higher pumping efficiency. Possible effect of divertor leakage was pointed out for both devices, where the complex 3D divertor geometry makes accurate alignment of target plates difficult. It was also noted that remote pumping needs to be considered in DEMO, and not as it is done in the present-day devices with pumps installed directly under the divertor structure. Importance of He-pumping was also mentioned, where very few experiments exist in both devices, and thus 
require further assessment. In order to predict divertor neutral pressure accurately and to design the pumping system in DEMO, improvement of transport codes is a prerequisite, as it will be a common issue for tokamaks.

Both devices have not suffered from significant core impurity accumulation so far. The $3 \mathrm{D}$ edge magnetic field structure is considered responsible for the edge impurity screening effects, where the friction force drives impurity towards the divertor region. Measurements in W7-X and LHD confirm the existence of such impurity flow directed toward the divertor plates. It was also mentioned that in $\mathrm{W} 7-\mathrm{X}$ and LHD, electron cyclotron resonance heating $(\mathrm{ECRH})$ is effective to avoid impurity accumulation in the core plasma. It was noted, however, that the screening is not perfect and finite impurity influx exists, which may not be visible in the pulse length of the present-day experiments but can be significant in long pulse or steady state operations. Therefore, studies on impurity transport and control in a core-edge coupled system especially for high $\mathrm{Z}$ impurity is important in future devices with steady state operation.

In W7-X divertor, heat load mitigation with detachment operation was very successful. A stable complete detachment (close to $100 \%$ radiation) with a 26 second pulse was demonstrated, where the divertor heat load was reduced by a factor of 10 with $Z_{\mathrm{eff}}<2$, while keeping high divertor neutral pressure sufficient for pumping. $\mathrm{N}$-seeding experiments showed easy detachment and feedback control. Ne-seeding experiments also showed stable detachment with significant radiation fraction from the confined region with slight increase of $Z_{\text {eff. }}$ These results hint the direction for W7-X stellarator reactor plasmas that may allow stable detached operation just with radiation from the SOL, but possibly with some radiation fraction from the edge of the confined plasma edge, if needed.

In the impurity seeding experiments of LHD, the general tendency was that $\mathrm{Ne}$ seeding provided heat load mitigation in all toroidal locations, while $\mathrm{N}$ seeding gave rise to strong toroidal asymmetry in the heat load distribution. K. Mukai (Japan) showed that sustainment of longer duration of the detached phase with impurity seeding was not very successful and it needs to be explored in the upcoming experiments, where impurity mixture seeding is being attempted. The RMP application is another scheme to mitigate the divertor heat load in LHD, in which stable detachment is realized. The divertor heat load was found to be modulated in accordance with the mode number of the RMP field. Some isotope effects were observed in hydrogen and deuterium plasmas, which needs to be investigated further in order to extrapolate to reactor plasmas.

In both devices, slight degradation of the core plasma performance was observed during the detachment operation $(<20 \%)$ depending on the scheme and impurity species. These results suggest that optimization of the island size or stochastic layer width and field line connection length with respect to radiation fraction, divertor neutral pressure and core plasma performance is a key issue in the stellarator-based DEMO.

Divertor heat load prediction towards DEMO is very important in order to optimize the divertor functions together with the whole system of the power plant. F. Warmer (Germany) and T. Goto (Japan) discussed the strategy and possibility of simple model development for heat load prediction in DEMO in a frame of system codes. EMC3EIRENE computations for W7-X was presented, where broadening of heat and particle deposition profiles on divertor plates are predicted due to both the longer connection length $(>1 \mathrm{~km})$ and to the higher density at the divertor region. The possibility of a high-density operation scenario was pointed out as an advantage of the stellarator without suffering from Greenwald density limit.

In summary, the following issues were raised as high priority to optimize the stellarator/heliotron divertor towards DEMO:

- Current pumping capability is not or not yet demonstrated in experiments especially in high density operation with detachment. The upcoming experiments in the next few years will address this issue with improved pumping systems.

- Impurity control for high-Z materials and He especially in long pulse operation, where infinitesimally small impurity influx, despite impurity screening, becomes visible. Development of core decontamination schemes, such as ECRH, and experiments with high-Z PFC are mandatory. Replacement of divertor target to $\mathrm{W}$ is ongoing in LHD and foreseen in W7-X. Long pulse discharges are planned in both devices in the next several years.

- For prediction of divertor heat load in DEMO, simple model development is undertaken in a frame of system code, focusing on the unique features of stellarator such as the long connection length and high-density operation.

\subsection{Alternative materials for PFCs}

This session (chaired by R. Neu) specifically dealt with the use of liquid metals (LM) as an alternative to solid plasma facing materials (PFMs).

Liquids potentially may be attractive because of: (i) the absence of embrittlement; (ii) their replenishing capabilities resulting in small armour thickness and thereby potentially higher heat load capability; and (iii) the possible resilience to transients because of the non-existence of persistent melt layers and enhanced vapour shielding as emergency running properties.

Potential drawbacks of liquids are: (i) unduly high erosion through evaporation/sputtering/chemical erosion resulting in too large plasma contamination leading to power losses through radiation and dilution; (ii) huge material migration 
Journal Title

posing operational/safety issues; and (iii) the potential excessive tritium retention. On the technical side, their implementation is complicated because of issues with the stability of free LM surfaces during transients and their integration into 'real PFCs', requiring at least one loop for LM and one for the cooling media, or even another loop for heating the LM.

The session comprised three oral contributions and three posters:

- E. Kolemen (United States) with a talk on the U.S. plans for "Fast flowing liquid metal divertor design options: Experimental and numerical studies";

- T. Morgan (Netherlands) with a talk on "Liquid metal conceptual divertor designs for the European DEMO";

- R. Goldston (United States) with a talk on "Analyses and experiments towards a lithium vapor box divertor";

- R. Neu (Germany) with a poster on "Behaviour of Tin under low-temperature deuterium plasma irradiation";

- V. Makhlai (Ukraine) with a poster on "Characterization of liquid metals as prospective divertor materials under transient plasma loads"; and

- A. Khodak (United States) with a poster on "Liquid metal modelling for plasma facing components".

Subsequently, the role of erosion and evaporation, as well as the consequences for the migration of the liquid metal and for the contamination of the core plasma, were discussed. Differently to present day machines, a future reactor will operate with hot walls far above the melting point of the envisaged metals, which will strongly change their deposition characteristic in the main chamber. Experiments in both FTU and EAST seem to show that no increase of $Z_{\text {eff }}$ occurs in the main chamber due to accumulation of Li. However, since Li has charge $3, Z_{\text {eff }}$ measurements are of limited significance in view of the plasma dilution. Liquid tin (Sn) is seen as a high$\mathrm{Z}$ alternative because of its lower erosion rate and vapour pressure. However, once $\mathrm{Sn}$ has migrated to the main chamber, its impact on the plasma performance could be much worse than that of $\mathrm{Li}$ due to its much higher radiation loss parameter. Concomitantly, the positive low vapour pressure of Sn could make accumulation avoidance in the main chamber more difficult to achieve. The planned divertor tokamak in Compass- $U$ foresees operation with hot walls and could therefore contribute significantly to the clarification of the above mentioned questions. Another ingredient in reducing the amount of LM in the main chamber could be the so-called vapour box. This concept relies on strong baffling and differential pumping of the Li vapour produced at the divertor targets. At the meeting, one of the conclusions was that substantial efforts should be devoted to the modelling of transport and migration of LM under realistic plasma and wall conditions.
Connected to the potentially high erosion and large migration, co-deposition of tritium could be an issue. This is important not only from the safety aspect but also from the operational point of view, because higher $\mathrm{T}$ inventories will increase (unnecessarily) the required breeding ratio.

Another very important topic for discussion was the technical readiness level (TRL) of the LM concept and the rate at which the LM PFC developments are progressing. Participants agreed that at the current $T R L \leq 3$ and rate of progress, it is unsure whether LM can be a viable back-up solution in a DEMO design. The LM divertor concepts are in fact many years behind in development if compared to the solid divertor targets. Soon, the community needs to decide whether to increase or to abandon the efforts on all levels of LM research and development. At the moment, several LM PFC mock-ups are under development (ENEA, EAST, NSTX). However, in addition to testing in high heat flux facilities and linear plasma devices, large scale testing in a divertor tokamak with substantial heat load will be necessary to provide confidence in the concept. Specifically, the behaviour of LM PFCs with impurity seeded plasmas (for example nitrogen seeding is not an option when using $\mathrm{Li}$ ), under transient loads and in a changing local magnetic field, must be addressed.

In support of the presented investigations, IAEA has initiated a Coordinated Research Project (CRP) on 'Vapor Shielding', which aims at providing answers to some of the highlighted issues [12]. The CRP's short-term main activities comprise the calculations and modelling of various processes with plasma species (e, H/D/T, He) and LM atoms ( $\mathrm{Li}, \mathrm{Sn}$ ) and molecules $\left(\mathrm{LiH}, \mathrm{Li}_{2}\right)$. Further, molecular dynamics (MD) simulations will be performed to model:

- Sputtering, retention, reflection of $\mathrm{D}$ and $\mathrm{D}_{2}$ of amorphous Li surfaces as well as fully deuterated Li surfaces;

- The evolution of surface layer chemistry and morphology upon evaporative deposition technique of $\mathrm{Li}$ and $\mathrm{LiO} 2$ on Mo substrate; and

- The solubility and sticking of $\mathrm{D}$ on $\mathrm{Li}, \mathrm{LiD}$ and $\mathrm{LiO}_{2}$ surfaces.

In support of modelling, EBIT experiments will be performed to acquire Sn spectroscopic data. Additionally, all LM divertor target material candidates $(\mathrm{Li}, \mathrm{Sn}$, and $\mathrm{LiSn}$ mixtures $(\mathrm{Li} 80 \% \mathrm{Sn} 20 \%))$ will be irradiated with $10-20 \mathrm{MW} / \mathrm{m}^{2}$ in the neutral beam facility at CIEMAT, comprising also simultaneous high-power laser irradiation to study the effect of ELMs.

\subsection{Core-boundary plasma compatibility}

This session (chaired by A. Leonard) began with a summary by A. Leonard on core plasma, particularly edge $\mathrm{H}$ - 
mode pedestal, issues that will constrain the design future divertors. The edge pedestal constrains the divertor design in the sense that it sets the upstream boundary conditions for the SOL and divertor plasma. The presentations and discussion in this session focused on four of these boundary conditions: (i) heat flux density flowing in the SOL towards the divertor; (ii) upstream separatrix density; (iii) upstream seeded impurity density; and (iv) sensitivity of the X-point region to cooling and strong radiative energy dissipation. The divertor plasma conditions, particularly heat flux dissipation depends nonlinearly on these parameters and will thus significantly constrain divertor design for a given future core plasma scenario. Without an accurate prediction of these parameters, design of future divertors will be very uncertain.

The heat flux width in existing tokamaks is well described by a plasma drift model [13], and scales as $\lambda_{\mathrm{q}} \propto B_{\mathrm{p}}^{-1}$ (where $B_{\mathrm{p}}$ is the poloidal field at the outboard midplane), without a size dependence. However, theory and modelling suggest that in future larger high field tokamaks this scaling may be exceeded, reducing the challenge of heat flux control to less than might otherwise be expected $[14,15]$. C.S. Chang (United States) presented results from the XGC code which predicts the critical parameter for turbulence to expand the SOL beyond the drift width to be $B_{\mathrm{pol}} a \rho_{\mathrm{i} \text {,pol }}$, where $B_{\mathrm{pol}}$ is the outboard midplane poloidal field, $a$ is the minor radius and $\rho_{\mathrm{i}, \text { pol }}$ is the outer midplane poloidal ion gyro-radius. This would result in a factor of several increase in heat flux width for ITER. Simulations presented by X. Xu (United States) using the BOUT ++ code support similar conclusions, though in the BOUT ++ case, the turbulence was primarily driven by electromagnetic instabilities, and in the XGC case, electrostatic turbulence was primarily driven by trapped electron modes. In both cases, with larger size the turbulent growth rate in the H-mode pedestal is expected to exceed the shearing rate and therefore resulting in increased radial transport and heat flux width when compared to the suppressed turbulence in the pedestal of existing devices.

Another mechanism for heat flux width expansion due to MHD ballooning stability was also summarized by A. Leonard. Data from AUG, JET and DIII-D demonstrate that the midplane separatrix pressure gradient increases with density until it saturates at the MHD ballooning stability limit, when the separatrix density reaches half/of the Greenwald density [16]. Pushing the density beyond this limit results in broadening of the SOL and heat flux width. The implication for future tokamaks is similar to that of predictions of increased SOL turbulence described above in that divertor dissipation, and detachment may be achievable at lower upstream density with greater compatibility in case of core operational scenarios. The implications of increased pedestal and SOL turbulence on pedestal as well as on core performance are still to be studied.
S. Mordijck (United States) described experimental work on pedestal density transport, focusing on recent coordinated analysis efforts in the United States. Pedestal density transport is a critical issue for divertor design; indeed, it sets the upstream separatrix density that will be compatible with a given core scenario density. In existing devices, the pedestal is fuelled primarily from divertor recycled neutrals with the pedestal density gradient increasing at the higher,SOL density required for divertor dissipation. In future pilot plant scale tokamaks, recycled neutrals are expected to be largely ionized in the divertor and SOL, with the core and pedestal only fuelled by central sources such as injected fuel pellets. The effect of reduced fuelling on the pedestal density structure and the relationship between separatrix and pedestal top density in future large tokamaks is uncertain due to poor understanding of the underlying pedestal particle transport. However, dedicated comparisons between DIII-D and Alcator C-Mod suggest that a pedestal pinch may preserve a significant pedestal density gradient, even in the absence of core fuelling in future devices. This uncertainty calls for increased international efforts, both experimentally and theoretically, to develop a predictive model of edge pedestal transport. A significant aspect of this effort will be diagnostic and interpretive analysis development to determine the core and pedestal ionization source profile in existing experiments.

S. Henderson (United Kingdom) described experiments and data analysis on AUG to quantify and scale the nitrogen seeding density required to achieve divertor dissipation and detachment [17]. Nitrogen concentration values obtained from spectroscopy were in line with estimates from seeding rates and deuterium fluxes from neutral pressure measurements. The scaling of nitrogen concentration required for divertor detachment scaled with upstream density and input power roughly as expected from simple scaling arguments, $c_{\mathrm{z}} \propto P_{\mathrm{sep}} / B_{\mathrm{p}} f_{\mathrm{GW}}^{2}$ [18], but at somewhat lower absolute magnitude. This work provides a basis for examining the relationship between seeded impurity densities required for divertor dissipation and the level of seeding that can be tolerated by the core plasma. It was concluded that future work should examine seeded impurity compression in the divertor, the ratio between upstream and downstream concentration, and its dependence on such factors as power, plasma SOL flow and SOL broadening due to turbulence.

A presentation from A. Jaervinen (United States) summarized the challenges of integrating dissipative divertor operation with high performance discharges in DIII-D. A key feature is to develop edge pedestal scenarios that remain in the peeling regime of pedestal stability at high density. While future large tokamaks may inherently operate in this regime, Super H-mode [19] was exploited to examine this regime in an existing, smaller tokamak. With nitrogen seeding, high performance was maintained while increasing divertor radiation and reducing the divertor target $T_{\mathrm{e}}$ to less or equal to 
Journal Title

$15 \mathrm{eV}$. Higher seeding rates resulted in a radiative collapse of the X-point with significant reduction in pedestal and core performance. Core radiation was increased with seeding of higher-Z impurities, $\mathrm{Ne}$ and $\mathrm{Ar}$, but the total radiation was limited by the onset of core MHD activity, neoclassical tearing modes (NTMs). The MHD instability onset occurred for values of $P_{\text {sep }}$ several times above the L-H power threshold. This result indicated an additional challenge for use of edge mantle radiation for divertor heat flux control. The presentation also highlighted how excessive low- $Z$ impurity seeding can lead to radiative collapse of the X-point region and subsequent reduction of the pedestal pressure and core confinement in advanced tokamak scenarios. This contrasts with previously presented AUG data where nitrogen seeding produced a strongly radiating X-point reduced pedestal pressure, but with adequate core confinement due to profile peaking [20]. As a result, further work with the pedestal community to identify attractive pedestal regimes and to develop the appropriate physics understanding was called for.

The discussion following the presentations in this session centred around the topics of heat flux width scaling and pedestal density transport. Discussion on heat flux width scaling focused on interpretation of the modelling results predicting a broader heat flux in future larger devices than would be expected from empirical scaling from existing devices. While the $\mathrm{XGC}$ and $\mathrm{BOUT}++$ simulations reproduce the empirical scaling in existing devices, they both predict significant broadening of the heat flux width in ITER compared to the empirical scaling. They both find the radial heat transport in the SOL is dominated by plasma drift physics in existing scale tokamaks, but turbulence originating in the pedestal gradients dominates in future larger devices. The discussion identified the challenge of validating the turbulence physics in the simulations with data from tokamaks where the radial transport is still dominated by drift physics. A further complication is that the two simulation codes find different instabilities, electromagnetic versus electrostatic, responsible for the transport. Also, it was pointed out that these simulations require extensive computer resources making it a challenge to carry out extensive modelling scans. These required computational resources also prove a challenge to self-consistently model the pedestal gradients that drive the turbulence. It was pointed out that regimes in existing devices with more turbulent pedestals, such as wide-pedestal QHmode or Grassy-ELM regimes, could be a productive platform for such validation studies.

Discussion on pedestal density transport noted the importance of capability to project the $n_{\text {sep }} / n_{\text {ped }}$ ratio, as $n_{\text {ped }}$ is typically defined by the operational scenario and divertor heat flux dissipation is nonlinearly dependent on $n_{\text {sep. }}$. Projection of this ratio to future devices is challenging because the radial particle flux through the pedestal is dominated by edge recycling in existing devices but is expected to be much smaller in future larger devices where a smaller radial particle flux will be supplied by central fuelling sources such as pellets. Discussion pointed out that future tokamaks will be primarily fuelled through pellet injection and therefore pedestal particle transport research should examine regimes in existing tokamaks with strong pellet fuelling. For progress in developing predictive capability for the pedestal density profile, an increased effort was called for in measuring the pedestal and core neutral fuelling source, and resulting radial ion flux, for testing emerging models of pedestal transport. Measurement of neutral fuelling will need significant additional resources for both diagnostics and the interpretive modelling required to determine inherently two-dimensional (2D) profiles. It was also pointed out that pedestal fuelling is usually dominant in the X-point region, and therefore theory and modelling should also start incorporating strong poloidal asymmetries in the fuelling profile.

\subsection{DEMO divertors design}

This session (chaired by M. Wischmeier) dealt with selection of design criteria for a device that would follow ITER, often referred to as DEMO, which aims to demonstrate fusion power plant performance.

For designing a DEMO facility, the integration of several design constraints needs to be accomplished and a separate IAEA workshop deals with the overall issues of the DEMO design itself [21]. In this meeting, the aspects of integrating possible power and particle exhaust solutions and their impact on the design specification were discussed.

The first presentation was a summary of EU DEMO design efforts given by M. Siccino (EUROfusion). This presentation described the integrated modelling approach used to link the core plasma specifications with the divertor design. The work highlighted the particular importance of the dynamics of transients, such as pellet injection for fuelling, and how they must be accounted for in the divertor design. Results from M. Wade's (United States) systems code analysis on power exhaust requirement for future fusion power systems were presented by A. Leonard (United States). The goal of this effort was the optimization of a pilot plant at the lowest capital cost. This design optimization found that the total cost is not sensitive to the divertor physics assumptions if the requirements for heat flux mitigation are not too severe. Also, improvements to core confinement relax the divertor design requirements. N. Asakura (Japan) presented results from the Japanese DEMO divertor design studies. Here, a credible design for divertor heat flux control was found with argon injection for radiative dissipation without the need for an advanced configuration. A critical issue of control of the detachment front was identified. Finally, F. Militello (United Kingdom) presented an analysis of advanced divertor configurations for application to DEMO. This work highlighted potential benefits, but also engineering design 
constraints and trade-offs as well as the difficulty of magnetic control of these configurations.

Additional supporting material was provided in an accompanying poster session, including:

- H. Du (China) with "A possible divertor combined the advantages of supper-X and snowflake for CFETR/DEMO";

- Y. Igitkhanov (Germany) with "Assessment of the pumping efficiency in DEMO conventional and alternative divertor configurations";

- R. Ambrosino (Italy) with "Electromagnetic and mechanical analysis of alternative magnetic divertor configurations for DEMO";

- R. Kembleton (United Kingdom) with "Engineering integration constraints for advanced magnetic divertor configurations in DEMO";

- L. Xiang (United Kingdom) with "First multi-fluid modelling results of super-X divertor in DEMO with $\mathrm{Ar}$ seeding";

- S. Kahn (United Kingdom) with "Impact of impurity seedings for divertor protection against intolerable heat loads and tungsten sputtering on general on plasma performances using the SYCOMORE system code";

- N. Asakura (Japan) with "Progress of divertor design concept for Japanese DEMO";

- M. Umansky with (United States) with "Scoping study of dissipative divertor scenarios for SPARC";

- S. Mao (China) with "Simulation study of the radiative divertor of different seeded impurity species for CFETR";

- S. Muldrew (United Kingdom) with "Status of Divertor/SOL modelling in PROCESS".

During the discussion session, an aspect raised was to which degree plasma material interaction drives the overall plasma solution. Here, the mutual interdependency of the SOL, the pedestal and core physics plays a crucial role and thus this discussion session overlapped (to some degree) with that on core-boundary plasma compatibility (Section 2.6), due to the recurring points of debate. However, an overall theme for this session was that for developing design criteria, not only the steady state average solution of the power exhaust problem is an important topic for designing DEMO but also to the dynamics of the system plays a crucial role due to the size and forces of the coils in such a device. The risk from ELMs needs to be further assessed, and ELMs may not be tolerable at all or only to a small degree, i.e. less than a percent of the pedestal pressure. A question which remains open is how to assess that in existing devices as this relates to the question of the "burn through" of ELMs in detached divertors. Another aspect raised was how to mitigate the risk of disruptions for the PFCs if such events appeared despite all the efforts to avoid them. Mitigating the risk of a re-attaching divertor plasma was a further risk discussed, and one of the proposed options was sweeping the strike points over large amplitudes. This requirement impacts the selection of the divertor geometry and the required resilience with respect to forces of the coils systems. The required resilience of the coil system to forces induced by variations of the magnetic equilibrium was also an aspect discussed in the context of investigating alternative divertor configurations (ADCs) as possible power exhaust solutions for a DEMO device. Such requirements restrict the range of possible ADCs and so does the accessibility to replace divertor modules as a function of positioning of required poloidal field coils. In fact, control is difficult for all $\mathrm{ADC}$ configurations and the engineering aspect looks challenging where the physics appears appealing. Nevertheless, also from the physics point of view, the capability of modelling small power fall-off length regimes with turbulence as well as with transport codes remains extremely challenging in large systems. The role of the size of the machine was raised as an important aspect in all of the discussions and so was the possibility to extrapolate observations from/today's devices to DEMO in view of the pumping efficiency and the baffling of neutrals. It was pointed out that a major difference between existing devices and DEMO is the ratio of the mean free path of neutrals to the size of the divertor, which is also connected to the questions on divertor closure for neutrals observed on today's devices as a function of divertor geometry or magnetic geometry. These questions ultimately may also impact the relation of divertor detachment and confinement. ITER in this respect is seen as a crucial experiment that may highlight major differences to conclusions drawn from today's devices. It was further noticed that possibly some representations from system codes need to be solicited for a future meeting as DEMO design points are defined using such codes and the possible exhaust solution enters these codes as a parameter. However, the dynamics of the exhaust solution appears to be only in its very early stages. Here, the question on the role and the availability of reduced models arose, which could then also be used in system codes. All these items converged in a key question which was brought up as a further point of discussion on how to reliably extrapolate from operating regimes of existing devices to DEMO prior to the operation of ITER. Most of the relevant DEMO exhaust scenarios assumed solid PFC solutions. A question that was thought it needs to be addressed was how plasma scenarios may differ when assuming liquid metal PFC solutions for DEMO.

Finally, the role of the competition between making the solution work and making it economically attractive was only marginally addressed at this meeting. 
Journal Title

\subsection{Divertors in next step devices}

This session (chaired by N. Asakura) began with a review on progress in operation space study and lifetime issues of the ITER divertor by R. Pitts (ITER Organization). The ITER first divertor will be used until the first deuterium-tritium (DT) operation campaign ( $\sim 13$ years). Impurity seeding with relatively low-Z such as $\mathrm{N}$ or $\mathrm{Ne}$ is a baseline scenario of the power exhaust, and operation space of the peak heat load $\left(q_{\text {target }}<10 \mathrm{MW} / \mathrm{m}^{2}\right)$ in the partial detachment was determined with sensitivities of key parameters. These studies pointed out common properties for the conventional divertor design, and they are an important simulation database for extrapolation to DEMO. Confirmation in existing and future experiments will be required.

Scaling of the peak $q_{\text {target }}$ in ITER as a function of the neutral pressure near the divertor pumping slot $\left(p_{\mathrm{n}}\right)$, i.e. $q_{\text {target }}$ reduction with $p_{\mathrm{n}}$ (from $1.5 \mathrm{~Pa}$ to $\sim 10 \mathrm{~Pa}$ ), was shown as a common property in the closed divertor geometry. The total radiation power $\left(P_{\mathrm{rad}}{ }^{\mathrm{div}}+P_{\mathrm{rad}}{ }^{\mathrm{sol}}\right)$ was also increased similarly with $\mathrm{p}_{\mathrm{n}}$, while $P_{\mathrm{rad}}{ }^{\mathrm{div}}$ was mostly dominated at $80-90 \%$ of $P_{\text {rad }}{ }^{\text {div }}+P_{\text {rad }}{ }^{\text {sol }}$ in Ne seeding. $P_{\text {rad }}{ }^{\text {div }}$ in $\mathrm{N}_{2}$ seeding was more dominant and localized near the target. SOL plasma density $\left(n_{\mathrm{e}}^{\mathrm{sep}}\right)$ was reduced with increasing impurity concentration $\left(n_{\mathrm{imp}} / \mathrm{n}_{\mathrm{e}}\right)$ by the seeding, and $n_{\mathrm{e}}{ }^{\text {sep }}$ was comparable for each $n_{\mathrm{imp}} / \mathrm{n}_{\mathrm{e}}$ case over the $p_{\mathrm{n}}$ range.

From the viewpoint of engineering design, the peak $q_{\text {target }}$ at the shaped monoblock target was increased and the magnitude depended on geometry of the target and magnetic field such as the poloidal target angle, magnetic flux expansion and monoblock-gap. From the viewpoint of physics design, the peak $q_{\text {target }}$ at the outer target was increased by effect of the $\mathrm{E} \times \mathrm{B}$ plasma drift. On the other hand, the drift effect on radiation and impurity retention will be reduced with increasing the divertor size, compared to existing experiments. The effect of $\chi$ and $D$ reduction on the peak $q_{\text {target }}$ was reported before.

Criteria for the lifetime of $\mathrm{W}$-monoblock surfaces were reported. The local peak $q_{\text {target }}$ has so far been restricted by $16 \mathrm{MW} / \mathrm{m}^{2}$, since $\mathrm{W}$-recrystallization becomes dominant on the surface (to the depth of $\sim 2 \mathrm{~mm}$ ) for the long DT operation period of $\sim 2200$ hours $\left(7.9 \times 10^{6} \mathrm{~s}\right)$, even at the temperature lower than $1200{ }^{\circ} \mathrm{C}$. Other transient events such as attached plasma operation, failure of ELM and disruption mitigation were not discussed.

A DEMO divertor design study for the Chinese Fusion Engineering Testing Reactor (CFETR) was presented by R. Ding (China) and showed recent simulation results in the ITER-like closed divertor geometry for the steady state DEMO design with $P_{\text {fusion }} \sim 1 \mathrm{GW}, \mathrm{R}_{\mathrm{p}} / \mathrm{a}_{\mathrm{p}}=7.2 / 2.2 \mathrm{~m}$, and $P_{\text {sep }} \sim 200 \mathrm{MW}$. High power handling of $P_{\text {sep }} / R_{\mathrm{p}} \sim 28 \mathrm{MW} / \mathrm{m}$ and the long divertor leg of $1.7 \mathrm{~m}$ were similar to those of the Japanese DEMO(JA-DEMO) divertor concept in the previous session, and Ne seeding was used as the reference case. The efolding length of $q_{/ /}$profile near the SOL separatrix $\left(\lambda_{\mathrm{q} / /} \sim 4 \mathrm{~mm}\right)$ was slightly larger than ITER, assuming the same $\chi\left(=1 \mathrm{~m}^{2} / \mathrm{s}\right)$ and $D\left(=0.3 \mathrm{~m}^{2} / \mathrm{s}\right)$. Partial detachment was seen in both inner and outer divertors, and the peak $q_{\text {target }}$ was $\sim 5 \mathrm{MW} / \mathrm{m}^{2}$ at both targets, where radiation power was the dominant component of the peak $q_{\text {target }}$ rather than the plasma transport due to radiation zone localized near the target. The similar divertor geometry with a longer leg of $2.4 \mathrm{~m}$ was also studied with the detachment more efficient. Lifetime of Wmonoblock by the surface erosion was simulated by DIVIMP, and it survived for 3 years with 0.5 duty cycle operation. More systematic study of the geometry (length and shape) was planned.

J. Canik (United States) presented Compact Pilot Plant (CPP) concepts with the aim to produce electricity from fusion at the lowest possible capital cost. Development of high confinement and high beta plasma is one concept, and high magnetic field tokamak design is another one. The SPARC concept proposed high $B_{\mathrm{T}}\left(\sim 12 \mathrm{~T}\right.$ at $\left.R_{\mathrm{p}}=1.78 \mathrm{~m}\right)$, producing $P_{\text {fusion }}>50 \mathrm{MW}(\mathrm{Q}>2)$. Development of the power exhaust scenario in the main plasma (steady state operation) is the high priority challenge, which requires high confinement $\left(H_{98 \mathrm{y} 2}=1.5-1.8\right)$ and $\beta_{\mathrm{N}}=4-5$ as well as large power exhaust at high $n_{\mathrm{e}}$, i.e. large radiation fraction in order to not reduce $P_{\text {sep }} / P_{\text {LHth }}$ to less than the ITER-level $(<1.2)$. This is a significant challenge, compared to the conventional power exhaust scenarios for the DEMO designs. At the same time, control (heating, current drive, momentum, impurity etc.) of the high-performance plasma is another challenge for steady state plasma operation. Power exhaust concepts for high $P_{\text {sep }} / R$ and magnetic geometry optimization (conventional double null, long leg, and advanced magnetic configurations) will be determined in existing and future experiments such as a divertor upgrade of AUG, Divertor Tokamak Test (DTT) facility and MAST-U.

Power exhaust studies of the conventional and alternative divertors in the Italian DTT facility were summarized by N. Vianello (Italy). This facility will be built in Frascati, Italy with $R_{\mathrm{p}} / a_{\mathrm{p}}=2.14 / 0.65 \mathrm{~m}, I_{\mathrm{p}}=5.5 \mathrm{MA}, B_{\mathrm{t}}=6 \mathrm{~T}$, to optimize the divertor candidate of ITER-level power exhaust of $P_{\text {sep }} / R(=15 \mathrm{MW} / \mathrm{m})$. Simulations of the various magnetic configurations such as double null, $\mathrm{X}$-divertor, snowflake (SF), Super-X and negative triangularity were performed as a function of $P_{\text {sep }}$ with $\lambda_{\mathrm{q} / / \sim 3} \mathrm{~mm}, \quad n_{\mathrm{e}}$ sep $/ n^{\mathrm{GW}} \sim 0.12 / 0.25$ $\left(n_{\mathrm{e}}^{\mathrm{sep}}=0.5 /\left(1 \times 10^{20}\right) \mathrm{m}^{-3}\right)$, and seeding impurities of $\mathrm{Ne}$ or Ar. For the pure D puff, SF divertor was able to reach the detachment at higher $P_{\text {sep }}(=10-20 \mathrm{MW})$. For impurity seeding with high $P_{\text {sep }}(=36 \mathrm{MW})$ and $n_{\mathrm{e}}{ }^{\mathrm{sep}}\left(=10^{20} \mathrm{~m}^{-3}\right)$, detachment was obtained in all the configurations with high radiation fraction $(\sim 0.9)$ and reasonable $n_{\mathrm{Ar}} / n_{\mathrm{e}}\left(\mathrm{Z}_{\mathrm{eff}}=1.4-2.6\right)$. SF solution provided lower $n_{\mathrm{Ar}} / n_{\mathrm{e}}$ at the separatrix and $Z_{\mathrm{eff}}$ was deduced slightly for the Ne seeding. 
Physical design of the EAST lower tungsten divertor (conventional configuration) by SOLPS was summarized by C. Sang (China). The study examined Ar and Ne seeding impurities with $P_{\text {sep }}=4 \mathrm{MW}$. Location of the impurity injection from the SOL was more efficient than that from the private flux region. Ar seeding was efficient to produce the detachment, but $\mathrm{W}$ sputtering and $\mathrm{W}$ concentration in the main plasma were enhanced compared to those in Ne seeding (DIVIMP). In the future, the performance will also be assessed in quasi-SF divertor configuration.

Discussion began with detachment modelling, particularly focusing on electron temperature. Improvement of the detachment modelling is one of long standing issues for divertor design. The plasma pressure drop near the strike point, as shown by experimental evidence, should be simulated by the improvements of many process models. Introduction of "elastic collision with molecules" is one candidate to reduce $T_{\mathrm{e}}$ to less than $1 \mathrm{eV}$. Other candidates such as modelling of volume recombination and molecular activated recombination (MAR), photon transport model, and drift effects need to be studied. Reduction in $T_{\mathrm{e}}$ to less than $\sim 5$ $\mathrm{eV}$ in the attached region will be necessary to minimize net erosion by seeded impurities such as Ar. This will be required for the long operational period in DEMO and may become a lifetime issue in ITER. This needs to become a focused requirement for the divertor design. Accurate modelling and experiment database will be required, while self-consistent plasma modelling with $\mathrm{W}$-transport remains difficult.

Modelling of energy and particle diffusion, particularly in the far SOL was discussed in this session. Issues of coreboundary plasma compatibility were also discussed. Particle diffusion in the far SOL ( $2^{\text {nd }}$ SOL) will affect heat and particle loadings on the first wall rather than the divertor since $70 \%$ of the total heat flux is to the divertor region. However, this physics in the far SOL affects design of the first wall location (increase), and it is potentially a significant problem for the tritium breeding blanket design in DEMO. Turbulent modelling will provide diffusion coefficients for fluid modelling of these issues.

Core-edge coupling, i.e. divertor heat load sensitivity to the pedestal structure is another long standing issue for divertor design. Since the DEMO edge and SOL plasmas are generally as low or lower collisionality than ITER, the ratio of $n_{\mathrm{e}}{ }^{\mathrm{sep}}$ and $n_{\mathrm{e}}{ }^{\text {ped }}$ and influence on the divertor performance should be studied in ITER for application to DEMO. The pedestal model in the system codes should be confirmed and/or improved in order to extend to the DEMO divertor design with data from next step devices. At the same time, SOL transport models in popular simulation codes will be investigated under the low collisional condition.

Furthermore, net erosion of the $\mathrm{W}$ target becomes dominant in the DEMO level long time operation as discussed earlier. A divertor design able to achieve the "pronounced" or full detachment in order to reduce $T_{\mathrm{e}}$ and $T_{\mathrm{i}}$ in the attached plasma region needs to be considered. At the same time, control of the detachment needs to be robust with respect to position of the strike point due to less diagnostics in for DEMO operation.

It was emphasized that code validation (in the detachment) against experiments but also code-to-code will be necessary, not only at single profile but also at systematic scan of key parameters. This needs to be a key goal for future next step devices.

Finally, there was a discussion of what will be learned from planned future devices for application to DEMO. ITER will provide experience and guidance on appropriate standard divertor configurations, leg length, effects of a baffle and dome, etc. In particular the role of system size will be provided by ITER. Information on the ITER DT performance including inductive and high-performance scenarios will be expected in 2035-2040. The second divertor will also be tested during this period. However, it is uncertain if "advanced divertor" concepts may be tested during this period for the design of DEMO. CFTER operation is still in the early stages of development so there was little discussion of its impact on DEMO design.

Divertor and power exhaust solutions for SPARC (and CPPs) are under investigation. The CCP concept assumes $H_{98 \mathrm{y} 2}>1.5$ and high radiation fraction in the main plasma, which has no experimental database, in particular, for the steady state operation. While a high radiation fraction was achieved with reversed shear operations in JT-60U with $H_{98 \mathrm{y} 2}=1.5$, the control of the steady state remained difficult. As a result, the plasma database with good core performance $\left(H_{98 \mathrm{y} 2}>1\right)$, high radiation fraction $\left(f_{\mathrm{rad}}>0.7-0.9\right)$ at the high $n_{\mathrm{e}} / n^{\mathrm{GW}}$ will be high priority issue particularly for the next step after ITER.

\section{Acknowledgements}

The authors wish to thank the support of the IAEA, the International Programme Advisory Committee and all participants of the meeting for their contributions and enormous amount of work done that has made this event successful.

\section{References}

[1] Technical Meeting on Divertor Concepts (Proc. of a Technical Meeting, Vienna, 2019) IAEA, Vienna (2019), https://nucleus.iaea.org/sites/fusionportal/Shared\%20Docume nts/DC\%202019\%20Material.pdf

[2] Technical Meeting on Divertor Concepts (Proc. of a Technical Meeting, Vienna, 2015), https://nucleus.iaea.org/sites/fusionportal/Technical\%20Meeti ng\%20Proceedings/1st $\% 20$ IAEA $\% 20 \mathrm{TM} \% 20$ on $\% 20$ Divertor $\%$ 20Concepts/Website/index.htm

[3] Technical Meeting on Divertor Concepts (Proc. of a Technical Meeting, Suzhou, 2017), 
https://nucleus.iaea.org/sites/fusionportal/Pages/Divertor\%20 Concepts/2017/Presentations.aspx

[4] 2001 Special issue on the IAEA Technical Committee Meeting on Divertor Concepts, Plasma Phys. Control. Fusion 44 (6)

[5] T.E. Evans et al 2005 J. Phys.: Conf. Ser. 7174

[6] Kirk A. et al 2012 Phys. Rev. Lett. 108255003

[7] Schmitz O. et al 2016 Nucl. Fusion $\underline{\mathbf{5 6} 066008}$

[8] Jakubowski M. et al 2009 Nucl. Fusion 49095013

[9] Frerichs H. et al 2016 Phys. Plasmas 23062517

[10] Lee H.H. et al 2017 Nucl. Mater. and Energy 12541.

[11] Lee H. H. et al 2nd IAEA Technical Meeting on Divertor Concepts (Suzhou, 2017), https://nucleus.iaea.org/sites/fusionportal/Shared\%20Docume nts/Divertor\%20Concepts/2017/15.11/Lee.pdf

[12] Heinola K. et al 2019 Atomic Data for Vapour Shielding in Fusion Devices, Summary Report of the First Research Coordination Meeting, IAEA, Vienna,

https://www-nds.iaea.org/publications/indc/indc-nds-0781.pdf

[13] Eich T. et al 2013 Nucl. Fusion 53093031

[14] Chang C.S. et al 2017 Nucl. Fusion $\underline{57116023}$

[15] Li Z. et al 2019 Nucl. Fusion 59046014

[16] Eich T. et al 2018 Nucl. Fusion $\mathbf{5 8 0 3 4 0 0 1}$

[17] Henderson S.S. et al 2018 Nucl. Fusion $\underline{\mathbf{5 8}} 016047$

[18] Reinke M.L. et al 2017 Nucl. Fusion 57034004

[19] Snyder P.B. et al 2019 Nucl. Fusion $\underline{\mathbf{5 9} 086017}$

[20] Reimold F. et al 2015 Nucl. Fusion 55033004

[21] International Atomic Energy Agency, Fusion Portal, Reports of DEMO Programme Workshop Series, https://nucleus.iaea.org/sites/fusionportal/Pages/DEMOReports.aspx 